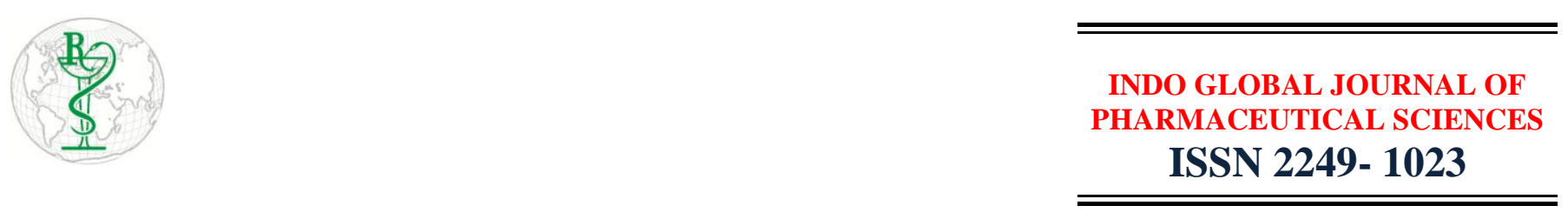

\title{
Can Food Supplements Combat Cancer: An Insight
}

\author{
Gupta $\mathrm{A}^{1}$, Vashist $\mathrm{N}^{2 *}$, Bajaj $\mathrm{S}^{3}$, Gahlot $\mathrm{V}^{2}$, Yadav $\mathrm{S}^{2}$, Maratha $\mathrm{S}^{2}$, Rajput $\mathrm{A}^{2}$ \\ ${ }^{1}$ OKC Division, Scientific Medical Research Ozone Pharmaceutical Ltd., Block A-3, Janak Puri, New Delhi-110058, India \\ ${ }^{2}$ SGT College of Pharmacy, Shri Guru Gobind Singh Tricentenary University, Gurugram, India \\ ${ }^{3}$ Delhi Institute of Pharmaceutical Sciences, New Delhi, India
}

Address for Correspondence: Vashist N, neelammsip@gmail.com

Received:

06.07.2018

Accepted:

30.09.2018

\section{Keywords}

Natural products;

Nutraceuticals;

Cancer;

Antioxidants;

Carcinogenic;

Anti-tumor.

\begin{abstract}
Cancer is an insidious disease affecting mankind in every country. The ability of cancer cells to migrate from one site of the body to other sites ("metastasis") is one of the biggest challenges in curing cancer. The present study brings new hope of future therapies to fight cancer. Designing an appropriate food to maintain proper health has become a necessity worldwide. To meet the requirement, the food industries are bringing the required changes in their products to fulfill the consumer needs. In recent years, many of the natural products are gaining popularity as nutraceuticals including dietary supplements and fortified foods that are marketed in a medicinal format such as powder, capsule or tablet in a prescribed dose. More rigorously, it is a designer food containing phytochemicals, biologically active compounds with a medical health benefit, including the prevention and treatment of chronic diseases. Natural products may be included either singly or in combination in the nutraceutical products for various purposes. They may be used to reduce the risk of various cancers. Now days, consumers are considering food not only for basic nutrition but for other health benefits also. Fruits and vegetables containing phytoconstituents are gaining popularity as potent anticarcinogenic agents such as allicin in garlic, isoflavones in soybeans, lycopene in tomato, alpha carotene in carrot, sulforaphane in broccoli and other glucosinolates in cruciferous plants. Cancer is one of the major deadly diseases and complexities of all diseases are such that no single available anticancer agents can satisfactory combat the multiple disorders of the disease. So there is an urgent need to search for better medicines for cancer. In this context, nutraceuticals are promising candidates to treat cancer. Therefore, this paper attempts to focus on promising therapeutic potential of nutraceuticals and highlight their chemical constituents in deadly disease like cancer. () 2019 iGlobal Research and Publishing Foundation. All rights reserved.
\end{abstract}

Cite this article as: Gupta, A.; Vashist, N.; Bajaj, S.; Gahlot, V.; Yadav, S.; Maratha, S.; Rajput, A. Can food supplements combat cancer: an insight. Indo Global J. Pharm. Sci., 2019; 9(1): 32-41. DOI: http://doi.org/10.35652/IGJPS.2019.9105.

\section{INTRODUCTION}

Cancer is an abnormal and purposeless multiplication of cells and tissues. It can invade distant tissues or even distant organs and results in death of the patient if it grows beyond that stage. Cancer may best be regarded as a group of neoplastic disorders characterized by:

(i) An abnormal growth of cells (ii) Ability to invade adjacent tissues and even distant organs (metastases)

(iii) Eventual death of the patient if the tumor has progressed beyond the stage when it can be successfully removed. 


\section{Indo Global Journal of Pharmaceutical Sciences, 2019; 9(1): 32-41}

Cancer is a multistage disease having the capacity to affect any tissue present at different locations of the body. The initiation \& progression of cancerous cells is discussed in Fig. 1. Cancers are generally classified into two major categories: solid tumors, leukemias and lymphomas. For solid tumours, these steps include: the formation of a mass of abnormal cells arising from the original changed cell; the development of new blood vessels to fuel the growth of the tumour cells; the invasion of this tumour into surrounding tissue; the shedding of tumour cells into the lymphatic system or bloodstream; and ultimately, the spread of these cells to other parts of the body (metastasis) where they can form additional tumours. Mutations in several critical genes can lead to tumors. Mutations in the tumor-suppressor gene p53 are found in about half of human tumors. The protein p53 guards a cell cycle check point that results in inactivation of p53 allows uncontrolled cell division.

A nutraceutical is a food that provides medical-health benefit in combination, to prevent and treat the disease. The term nutraceutical was introduced by Stephen DeFelice, MD, Founder and Chairman of the 'Foundation for Innovation in Medicine' in 1989 [1]. Such foods also commonly are referred to as functional foods, indicating they and/or their components may provide a health benefit beyond basic nutrition. Foods is the basic requirement of the body which provide nutrients, whereas nutraceuticals contain a health-promoting ingredient that shows a potential health benefit for the body [2]. "Functional" attributes of many traditional foods are reviewed with beneficial components in cancer. Etiology and the pathogenesis of cancer can be explained with the help of six hallmarks of cancer [3].

For so many of us, it is impossible to get adequate nutrition from the foods we buy in the grocery store. The fruits and vegetables are mostly harvested green, thus eliminating the valuable disease-preventing phytochemicals that are in abundance in vine ripened produce. Secondly, we live in a highly toxic environment, filled with pollutants and pesticides that throw off our body's ability to regulate it. Making a commitment to gradually include more vegetables in diet is the first step.

Certain vegetables having caretenoids as the cheif constituent e.g. asparagus, spinach, carrots, beets, winter squash, tomatoes, and sweet potatoes have low sugar content and are rich in fibres, having anti-cancer properties. The caretenoid rich vegetables also have antioxidants property which protects healthy cells from chemotherapy and radiation. The solubility of carotenoids increases with other fat soluble foods, inceasing their chances to enter the blood stream and making available for their action. Vegetables such as cabbage, broccoli, sprouts, and cauliflower have also been reported for their anti-cancer activity and body detoxification. Quercetin, allicin, S-allyl cysteine and selenium are the cheif constituets of onions and garlic. They help in fighiting prostate cancer and body detoxification.

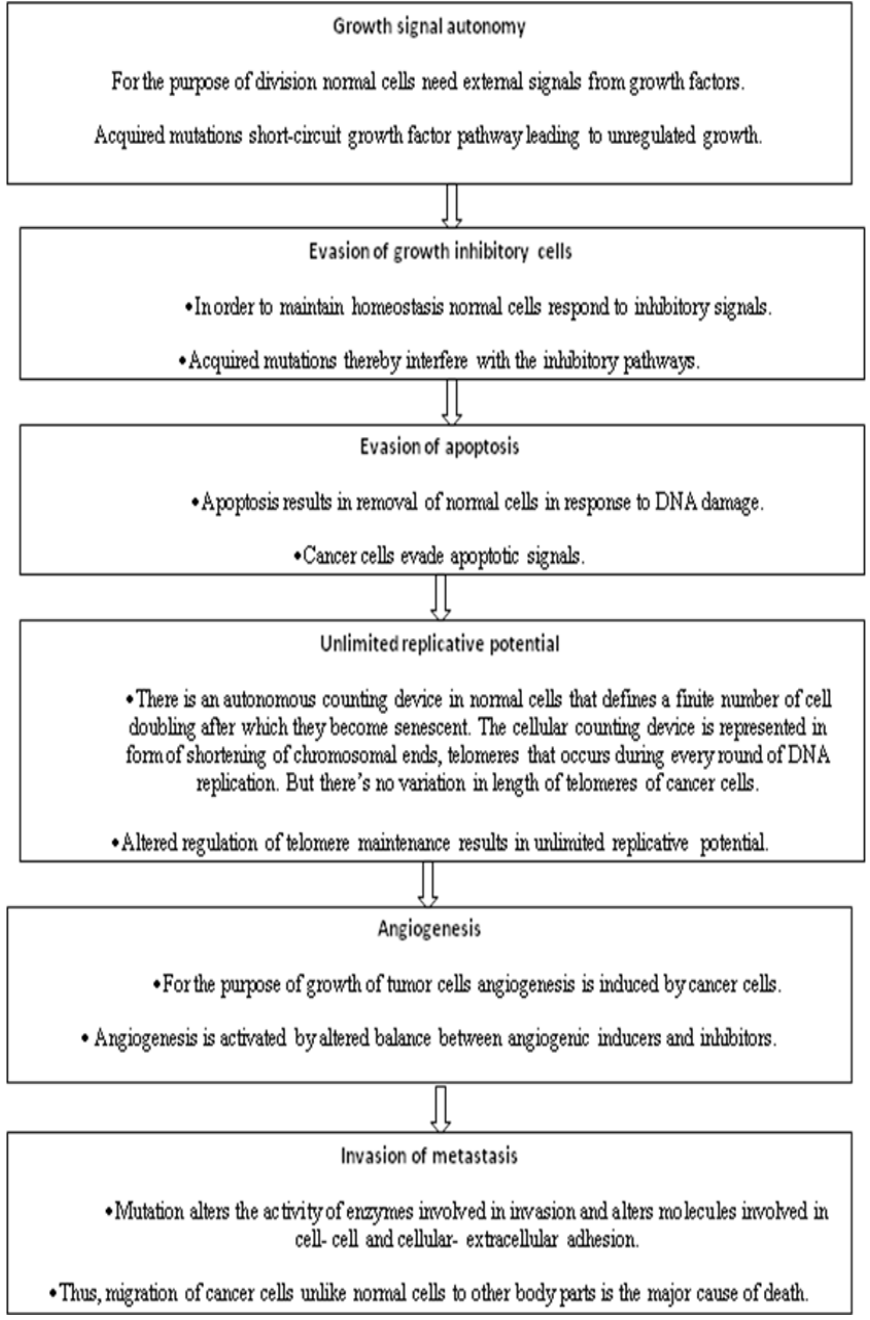

Fig.1. Stages showing the initiation \& progression of cancer cells in various tissues.

Adequate protein is vital for a healthy immune response to cancer and to rebuild any damaged tissue after surgery or treatment. Organic soya is a preferred source of protein, followed by beans, dried peas and lentils, nuts, pumpkin seeds, eggs and chicken and whole grains are the wonderful sources of vitamins, minerals and fibre. For variety brown rice, oats, barley, millet, amaranth, buckwheat and rye may be considered. High fibre grains are utilized greatly when the colon is maintained with sufficient microflora or friendly bacteria. Colorful berries contain a powerful agent ellagic acid, which triggers apoptosis in prostate cancer cells. This "self-destruction" mechanism is found in various berries like 


\section{Indo Global Journal of Pharmaceutical Sciences, 2019; 9(1): 32-41}

raspberries, strawberries, dark cherries, blueberries, cranberries and blackberries etc. Green tea has shown potential to prevent and prolong remission in cancer patients. Studies show that drinking green tea daily inhibits cell division and metastasis. The substances present in green tea such as catechin and epicatechin are polyphenolics have been known to act as antioxidants and immune stimulants. All cells in the body, even immune cells, need healthy fats and oils. Fat is the preferred fuel of muscle and it is a crucial component of cell membranes. Recently fish oil, olive oil, flax oil and even real butter are most recommended. All these prospects make nutraceuticals potentially useful in deadly chronic diseases like cancer [4].

\section{Herbal Products as hidden medical weapons}

Although modern medicines have become more influential and more specific for treating various conditions, there is a growing emphasis on the importance of diet, nutrition and lifestyle in preventing health problem and promoting well being. Herbal products were mankind's first medicine and they have served that function for centuries. In fact, most of the world's population is still treated with herbal medicines. Herbs may be included either singly or in combination in the nutraceutical products to reduce the risk of cancer.

\section{TRADITIONAL HERBS AND PLANTS WITH POTENTIAL}

Allin, Allicin, Allylic Sulfides, Organosulfides: Found in Garlic and Allium vegetables ginger and onions (Allium cepa) contain a number of compounds that have been associated with anti carcinogenic effect. The positive effect appears to be related to the presence of both oil- and water-soluble organosulfur compounds, primarily allyl derivatives such as allicin. Garlic is one of the oldest natural medicines known to mankind and can easily be included in recipes or taken as a tablet supplement. The active sulfur compounds may inhibit development of prostate tumors. Onions and garlic are powerhouses that contain substances like quercetin, allicin, Sallyl cysteine and selenium. They lower the incidence of esophageal, prostate, laryngeal, oral cavity, pharynx, breast, ovarian, prostate, renal, gastric and colorectal cancers. The anti-cancer action of garlic is produced by supressing cell cycle progression and inducing sucide of cancer cells or by decreasing the formation of new blood vessels providing blood flow to cancer cells and ultimately affecting carcinogen metabolism [5]. The active constituents of garlic have been reported to inhibit the chemical carcinogens during the initiation phase and promotion of carcinogenesis [6].

Alpha carotene: found in carrots, sweet potatoes, tomatoes and watermelons. These powerfully pigmented vegetables act as antioxidants, defensive healthy cells from chemotherapy and radiation [Fig 2]. Carotenoids are lipophillic, so more will be absorbed when taken with a meal or snack containing some healthy fat. In population studies, alpha carotene is related to reduced risk of lung cancer. It may slow down the proliferation of cancer cells. So carotenoids appear to play an anticancer role and increase immunity. Lycopene is increasingly gaining ground as the most powerful antioxidant in the carotenoids family, particularly in relation to prostate and breast cancer. Two carotenoids found in the eye are lutein and zeaxanthin, believed to protect against the leading cause of blindness in people over sixty five. Carotenoids also play an important role in keeping healthy heart; In LDL oxidation, antioxidants like vitamin $\mathrm{E}$, lycopene and $\beta$-carotene are consumed. Chopped vegetables in the presence of oxygen and sunlight makes them vulnerable for oxidation as contrast to the common beleif of heat resistant carotenoids. So prevent keeping the chopped vegetables in direct sunlight. Cooking foods slightly makes their carotenoids more readily available [7].

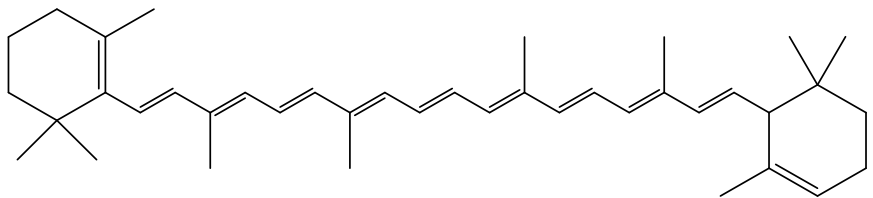

Fig.2 (1E,3E,5E,7E,9E,11E,13E,15E,17E)-3,7,12,16tetramethyl-1-(2,6,6-trimethyl-1-cyclohexenyl)-18-(2,6,6trimethyl-1-cyclohex-2-enyl)octadeca-1,3,5,7,9,11,13,15,17nonaene

Anthocyanins: Anthocyanins are hydrophillic vacuolar pigments and are red, purple, or blue according to $\mathrm{pH}$ and belongs to the class of flavonoids. Blueberries, blackberries, elderberries, strawberries, grapes, purple grapes and black currants are rich sources of anthocyanin .These phytochemicals helps in decreasing the probability of cancer occurence for pancreas, breast, esophageal, skin, colon and prostate cells. The anthocyanin-rich extracts (AREs) of commercially available fruits such as grape (Vitis vinifera), bilberry (Vaccinium myrtillus L.), and chokeberry (Aronia meloncarpa E.) were found to be having anti carcinogenic activity against colon cancer, the results had shown that all extracts inhibited the growth of coloncancer-derived HT-29cells and it was observed that chokeberry is the most potent inhibitor [8]. Anthocyanins also exhibit antioxidant properties which are credited to their phenolic structure. The in vitro antioxidant effects of anthocyanins were 


\section{Indo Global Journal of Pharmaceutical Sciences, 2019; 9(1): 32-41}

studied using several cell culture systems for colon, liver, breast and leukemic cells \& keratinocytes, and were found to be effective. It has been indicated that anthocyanins exhibited multiple anti-toxic and anti-carcinogenic effects. The effect includes directly scavenging reactive oxygen species, increasing the oxygen-radical absorbing capability of cells, enhancing the expression of Phase II detoxification enzymes, reduction in the formation of oxidative adducts in DNA, decreasing lipid peroxidation, preventing mutagenesis by environmental toxins and carcinogens, and decreasing cellular proliferation through modulation of signal transduction pathways [9].

Antioxidants: An antioxidant is a molecule having the capacity of inhibiting the oxidation of molecules found in fruits, vegetables, fishes, bread crust etc. It inhibits the oxidation by preventing reactive oxygen species such as superoxide anion, hydroxyl radical, singlet oxygen species and water. Anti-oxidants may prevent the cells from entering apoptosis or from committing suicide when they are in a very high oxidative state. Antioxidants are known to hinder the growth and reproduction in cancer cells, however oxidative stress may be responsible for the proliferation of cancer cells.

Resveratrol is one of the active constituent of red grape and act as an antioxidant. It's a phytoalexin produced naturally by several plants when attacked by certain pathogens viz. bacteria or fungi. Resveratrol, derived from Japanese knotweed, is produced and marketed as a nutritional supplement. As reported by Jang in 1997, its topical application prevented the skin cancer development in mice treated with a carcinogen, but no results of effects on human have been reported. Resveratrol acts by interfering stages of carcinogenesis such as initiation, promotion and progression. Many mechanisms in the pharmacological activity of resveratrol were indicated by the experiments carried out in cell cultures of varied types and isolated subcellular systems in vitro. These mechanisms include modulation of the transcription factor NF- $\mathrm{KB}$ (Nuclear factor Kappa B ) inhibition of the human cytochrome P450 1A1, alterations in androgenic actions and expression and activity of cyclooxygenase (COX) enzymes [10]. In vitro, resveratrol also inhibited the proliferation of human pancreatic cancer cell lines [11].

$\boldsymbol{\beta}$-Carotene: $\beta$-Carotene, a strongly-coloured red-orange pigment, belonging to the class of terpenoid family, is rich in plants and fruits. Carotene, inactive form of vitamin A, has $\beta$ rings at both ends [Fig. 3]. As a naturally occurring phytonutrient, some of the richest food sources of beta carotene are green, yellow, orange and red fruits and vegetables such as asparagus, broccoli, carrot, pumpkin, spinach, sweet potato, and watermelon. Beta Carotenes' possible benefits include reduced risk of cataract, coronary artery disease, and breast cancer and enhancing immunity for the elderly patients. Studies show that people who eat a meal contains rich in carotenoids, has got a much lesser risk of breast, colon cancer and lung cancer. Carotenoids fight against cancer cells through their antioxidant property and helps them to keep the body cells in proper communication as an effective precautionary step against the growth of cancer cells. From previous studies, it was found that consuming $\beta$-carotene may be effective in falling down the risk of prostate cancer in men who have low blood levels of $\beta$-carotene $[12,13]$.

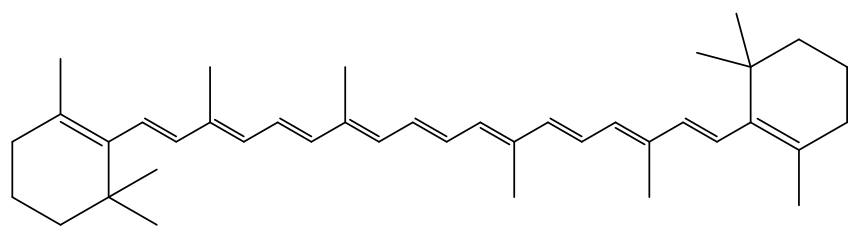

Fig.3. 1,1'-(3,7,12,16-Tetramethyl-1,3,5,7,9,11,13,15,17octadecanonaene-1,18-diyl)bis[2,6,6-trimethylcyclohexene]

Beta-sitosterol: $\beta$-Sitosterol, is chemically similar to cholesterol, belongs to the class of phytosterols family [Fig 4]. Sitosterols are white, waxy powders with a characteristic odor. They are hydrophobic and soluble in alcohols. They are abundantly found in beans, corn oil, nuts, peas, seeds, soybeans, wheat germ and whole grains. It is also used for strengthening the immune system as well as for preventing colon cancer, as well as for HIV/AIDS, cervical cancer, fibromyalgia. A study demonstrated that $\beta$-sitosterol decreases cancer cell growth by twenty four percent and induced programmed cell death by four timesusing prostate cancer cell line LNCaP (an androgen-dependent tumor) [14].

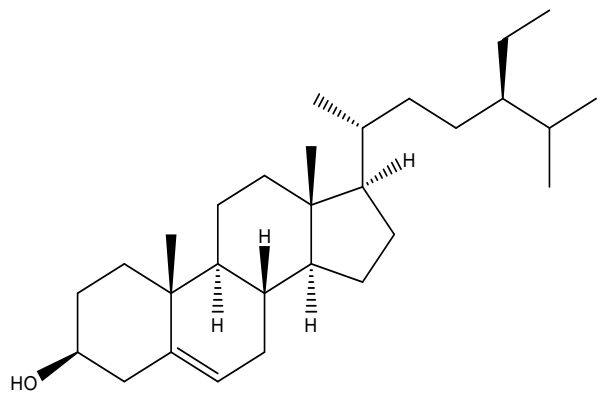

Fig.4. 17-(5-Ethyl-6-methylheptan-2-yl)-10,13-dimethyl-

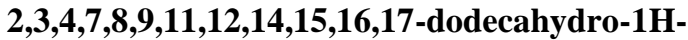
cyclopenta[a]phenanthren-3-ol.

Bioflavonoids: Citrin and Vitamin P are collectively known as Flavonoids (or bioflavonoids), a class of plant secondary metabolites. These are richly found in orange, tangerine, peach, pear, yellow pepper, grapefruit, pineapple, lemon, apricot, papaya and yellow raisin. They work with vitamin C to help in decreasing the risk of cancer, empower bones and teeth, heal wounds, keep skin healthy and lower the risk of 


\section{Indo Global Journal of Pharmaceutical Sciences, 2019; 9(1): 32-41}

heart attacks. By physiological processing of unwanted flavonoid, they helps to kill carcinogens and mutagens by socalled Phase II enzymes, and thus helpful in cancer prevention. Flavonoids could also persuade mechanisms that may destroy cancer cells and inhibit tumor invasion. It has been found that study participants who consumed foods containing specific flavonoids, like catechins found in strawberries, green and black teas; kaempferol from apples and brussel sprouts; and quercetin from onions, beans and apples, have reduced risk of lung cancer [15].

Caffeic acid and ferulic acid: Caffeic acid is a naturally occurring organic compound. It is found in all plants because it is involved in the biosynthesis of lignin, one of the principal sources of biomass. It is found in apples, asparagus, cabbage, grains, spinach, and tomatoes which are sources of cancer fighting agents. Caffeic acid (CA) and its derivative, Caffeic acid phenethyl ester (CAPE) have shown tumor-fighting properties. When CA and CAPE were administered orally and sub cutaneously it found a significant reduction in liver metastasis. These results confirmed and laid the base for the therapeutic potential of the compound. The capability of cancer cells to effect distant organs or tissues was found to be diminished by the use of CA and CAPE. Their effect was found through the selective suppression of MMP-9 enzyme activity and transcriptional down-regulation by the dual inhibition of NF- $\kappa \mathrm{B}$ (Nuclear factor Kappa B) as well as MMP-9( Matrix Metallopeptidase 9) was confirmed by catalytic activity [16].

Ferulic Acid is an organic compound, which is an abundant phenolic phytochemical found in components of plant cell wall such as arabinoxylans as covalent side chains.It possesess anti tumor - promoter, anti-inflammatory, anti-viral and arteriodilator properties. Ferulic acid may have direct antitumor activity against liver and breast cancers as reported by in vitro and in vivo studies. Ferulic acid may have proapoptotic effects in cancer cells, thus leading to their destruction. The cancer induced by exposure to the carcinogenic compounds such as benzopyrene and 4nitroquinoline 1-oxide may be prevented by Ferulic acid. Further alkylferulate, ferulic acid derivative has anticarcinogenic property. Anti-colon carcinogenesis activity was found in one of the Ferulic acid derivative, in which geranyl group is attached to the phenolic hydroxyl group of ethylferulate. The results of these studies may not be directly applicable to human use because these are not randomized controlled trials done with human participants [17].

Capsaicin: Capsaicin is the major pungent ingredient in red peppers. Reduces risk of colon, gastric and rectal cancers; inhibits tumor growth; destroys cancer causing substances. It is also a digestive aid, a topical pain reliever, and a valuable cancer-fighting compound [Fig 5]. Capsaicin is able to kill prostate cancer cells by producing them to undergo apoptosis as reported by American Association for Cancer Research .The studies were performed on tumors caused by human prostate cancer cell cultures developed in mouse models, and the results indicated that tumors treated with capsaicin were showed about one-fifth the size of the untreated tumors. Studies conducted on human volunteers in Japan and China has proved that natural capsaicin arrests the growth of cancer cells [18]. Capsaicin blocks the translocation of nuclear factor kappa B (NF-kB), activator protein 1 (AP-1), and signal transducer and activator of transcription (STAT3) signaling pathways that are required for carcinogenesis [19].

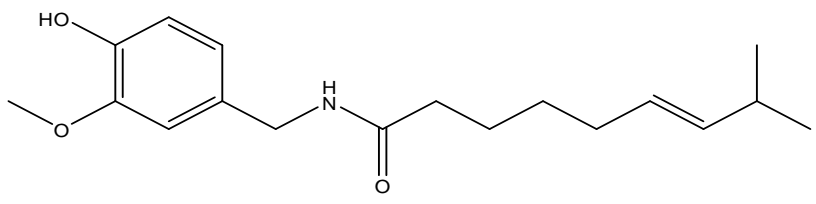

Fig.5. 8-Methyl-N-vanillyl-trans-6-nonenamide

Carnosol and Ursolic acid: Ursolic acid chemically is a pentacyclic triterpene acid, found its application in cosmetics. It is found in Rosemary and sage. Ursolic acid acts through glucocorticoid receptors and produces anti-cancer effect by blocking STAT3 activation pathway and human fibrosarcoma cells by falling the expression of matrix metal loproteinase- 9 [20]. Ursolic acid and carnosol are potent antioxidants that possess anti-tumor activity. Rosemary is a rich source of carnosol. The later possess a good antioxidant, antiinflammatory and anti-carcinogenic activity. It may also protect against skin and lung cancers. The creosol found in rosemary has demonstrated potent anticancer properties in animal studies. It increases the production of an enzyme that defends cellular DNA from being destroyed, and in one test appeared to have some success in offsetting the effects of a carcinogen known to cause breast cancer [21].

Catechin: Catechin is a polyphenolic, plant secondary metabolite. The term catechins is related to the family of flavonoids. Mostly found in apples, berries, black and green tea, chocolate, grapes and wine [Fig 6]. Potential benefits include increased immune function; decreased cholesterol production. Catechins are a powerful form of antioxidant that acts as an inhibitor of cancer cell growth. Catechins are hundred times more potent than vitamin $\mathrm{C}$ and twenty five times more powerful than vitamin $\mathrm{E}$ in their antioxidant/growth inhibitor properties. Flavonoids and catechins produce anti-cancer effect by inhibiting the progression of the cell cycle in cancerous cell and inhibit 
Indo Global Journal of Pharmaceutical Sciences, 2019; 9(1): 32-41

mutations that can leads to cancer. they have also shown their ability to protect cells from the harmful effects of $\mathrm{x}$-ray. Catechin \& epigallocatechin gallate (EGCG) produces anticancer effect in prostate gland by suppressing growth of tumors cells and also by inhibiting enzymes that help in the proliferation of cancer cells [22]. It has been reported that the esters of epigallocatechin gallate with docosahexanoic acid were found to suppress colon cancer in mice [23].

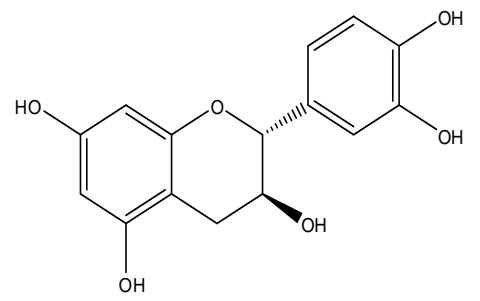

Fig.6. (2R,3S)-2-(3,4-dihydroxyphenyl)-3,4-dihydro-2Hchromene-3,5,7-triol

Curcumin: Curcumin is the chief constituent of Indian spice turmeric. It belongs to the ginger family (Zingiberaceae) [Fig 7]. Desmethoxycurcumin and bis-desmethoxycurcumin are two main other curcuminoids. The curcuminoids are polyphenols and are responsible for the yellow color of turmeric [24]. It's produces anti-cancer effect by inducing apoptosis in cancer cells without any cytotoxic effects on surrounding healthy cells. It has also been reported that curcumin produces anti-cancer effect by "modulating the growth of cancerous cells through regulation of many cell signaling pathways including cell proliferation pathway (cyclin D1, c-myc), caspase activation pathway (caspase-8, 3, 9), cell survival pathway (Bcl-2, Bcl-xL, cFLIP, XIAP, cIAP1), tumor suppressor pathway, death receptor pathway (DR4, DR5), mitochondrial pathway, and protein kinase pathway (JNK, Akt, and AMPK) etc. Because curcumin shows its effect through multiple cell signaling pathways, the likelihood of developing resistance to it is less [25]. Curcumin can also alter the activity of transcription factor NF$\kappa \mathrm{B}$,(Nuclear factor Kappa B) which has been associated to a number of inflammatory diseases such as cancer. It also helps to reduce activity of the enzymes called tyrosine kinase and protein kinase, which otherwise stimulate the growth and spread of cancer [26].

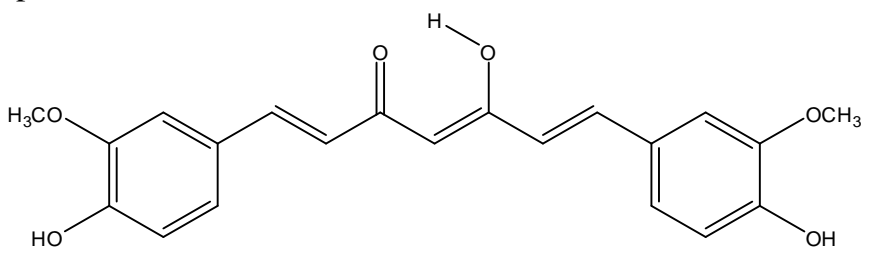

Fig. 7. (1E,6E)-1,7-bis (4-hydroxy-3-methoxyphenyl) - 1,6heptadiene- 3,5- dione
Dithiolethiones and Organosulfides: Dithiolethiones are a well-known class of cancer chemopreventive agents, found in cruciferous vegetables. Increased consumption of cruciferous vegetables such as broccoli, cabbage and brussels sprouts may reduce the risk of certain cancers, particularly breast cancer. Cruciferous vegetables such as garden cress, bok choy, broccoli and Brussels sprouts belonging to the family Brassicaceae (also called Cruciferae) constist of indoles, which is a unique class of phytonutrients that have proven themselves to regulate hormone levels, detoxify the intestines and liver and strengthen the body's immune system. They reduce risk of gastric, colon, and lung cancers, enhance immune system, inhibit tumor promotion, reduce cholesterol, lower blood pressure. The chemopreventive effects of dithiolethione compounds are attributed to their activation of antioxidant response elements (AREs) by reacting with the Nrf2/Keap1 [Nuclear factor (erythroid derived 2)] protein complex. ACS-1, a dithiolethione compound, inhibits multiplication of human cancer cell lines (A549 and MDAMB-231) by increasing the activity of the tumor suppressor protein phosphatase 2A (PP2A) [27]. In a study by a group of researchers reported a novel anticarcinogenic activity of an organosulfur compound from garlic, diallyl disulfide (DADS). Rodent demonstrations indicated significant inhibition of growth of H-rasoncogene transformed tumour [28].

Essential Fatty Acids (EFAs): EFAs are one of the main requirements of our body but human beings are not able to make them from other food components, so we must ingest them for good health. EFAs are found in fish, flaxseed oil, and walnuts. They help in reducing the symptoms of painful and frequent urgency to urinate in patients suffering from benign hypertrophy of prostate. They also improve the flow of blood to the penis and possibly may help to prevent the occurance \& progression of prostate cancer. Omega-3 fatty acids reduce prostate tumor growth, slow down histopathological progression, and increase survival [29]. Increased levels of docosahexaenoic acid, which is abundant in $n-3$ poly unsaturated fatty acid [omega-3] in red blood cell membranes, has been associated with a reduced risk of breast cancer [30].

Gingerol: It's a natural component of ginger and exhibits antiinflammatory and anti-tumorigenic activities [31]. According to the researchers leukotriene A4 hydrolase (LTA4H) protein might be a potential target for [6]-gingerol which diminishes anchorage-independent cancer cell growth by blocking LTA4H activity in HCT116 colorectal cancer cells. It indicates towards the significant role of [6]-gingerol in the prevention of colorectal cancer [32]. 


\section{Indo Global Journal of Pharmaceutical Sciences, 2019; 9(1): 32-41}

Isoflavones: Isoflavones are naturally occuring polyphenolic compounds belonging to the class of isoflavonoids. Some isoflavones and isoflavone-rich foods possess activity against certain types of breast and prostate cancer. These are generously found in soyabeans. Soya isoflavones has been associated with reducing the incidence of breast and prostate cancers. Genistein is the predominant isoflavone in soya, inhibits the growth of blood vessels. Tumors need to stimulate the growth of blood vessels to receive oxygen and nutrients. Without these new blood vessels tumors can't grow. Recent preliminary data suggest that soya may inhibit the growth of blood vessels in humans. Thus, iso flavones may actually be useful in the treatment of existing tumors. The activation of NF-kappa B and Akt signaling pathways which are responsible for the progression of cancer, are inhibited by genistein. Furthermore, genistein has been found to show potent inhibitor of angiogenesis and metastasis. Soya contains eight known classes of anti-cancer along with cell antiproliferation components. These include protease inhibitors, omega-3 fatty acids, phospholipids (lecithin), phytosterols, phenolic acids, saponins and isoflavones [33].

Limonene: Limonene is a colourless liquid hydrocarbon classified as a cyclic terpene possessing a strong smell of oranges. Lemon and orange oils contain limonene and geraniol also found in cumin. Limonene is derived from the essential oils of citrus fruits, mints, caraway, dill, lemongrass and other plants [Fig 8]. These components of lemon and orange oils have been shown to inhibit tumor growth. The mechanism of action of D-Limonene and its metabolites, perillic acid, uroterpenol, limonene1,2-diol and dihydroperillic acid is unknown but they may supress tumor growth through inhibition of p21-dependent signaling and apoptosis resulting from induction of the transforming growth factor $\beta$-signaling pathway [34]. Animal studies indicate activity of D-limonene against certain tumors like pancreatic, stomach, colon, skin, and liver cancers. Data also reported that D-limonene slows the promotion/progression stage of carcinogen-induced tumors in rats [35]. Because these oils are limited only in the peels of the fruit, researchers are looking at ways to include rind in filtered juices. Therefore, limonene may be moved to phase III trials for their establishment as anticancer nutraceuticals [36].

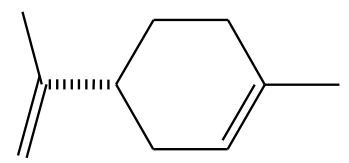

Fig.8. 1-methyl-4-(prop-1-en-2-yl)-cyclohexenes)

Phenolic Acid: Phenolic Acid is present in cauliflower, cabbage, garden cress, bok choy, broccoli, Brussels sprouts, tomatoes, celery, parsley, eggplant, peppers, soya, flaxseed, citrus, whole grains, cherries and berries. It fights against cancer through nitrosamine formation. The possible oncoprotective mechanisms of phenolic acids include antioxidant effects, steroid receptor binding, direct interaction with intracellular elements and signaling systems and aryl hydrocarbon receptor (AhR) binding and modification of subsequent signaling pathways [37].

Saponins: Saponins are naturally occuring glycosides found in legumes namely alfalfa, clover, peas, beans, chickpeas, lentils, lupin bean, mesquite, carob, soybeans, peanuts and tamarind. They exhibit various biological and pharmacological activity such as immunomodulatory, molluscicidal, antiviral, antiinflammatory, antitumor, antifungal, hypoglycemic, hypocholesterolemic. The suds in root beer is produced by plant biochemicals is an effective antimicrobial, cholesterollowering, and anticancer product. Digitalis, a saponin, used in heart ailments may also prevent cancer cells from multiplying by influencing the DNA in the cells [38].

Selenium: Selenium is present in trace quantity in our diet but is essential for the maintainence of antioxidant systems including glutathione peroxidase that neutralizes and protect against damage caused by reactive oxygen species and free radicals. It is present in nuts, grains, poultry, pumpkin seeds, and seafood. It is effective in the prevention of benign prostatic hyperplasia (BPH) and progression of heart disease. It may prevent oxidative damage to lipids, vitamins, hormones, and enzymes contributed in regular prostate functioning. Preliminary studies have reported that pumpkin seeds (high in selenium) may reduce hormonal harm to prostate cells thereby reducing the risk of developing prostate cancer. Data suggest its benefits in eliminating gastrointestinal and lung carcinomas $[39,40]$.

Squalene: Squalene, naturaly occuring organic compound, is obtained from shark liver oil for commercial purposes. Squalene is also present in vegetable oils obtained from amaranth seed, rice bran, wheat germ, and olives [Fig.9]. It is demonstrated that squalene produces inhibitory effect on chemically-induced colon, lung and skin tumour genesis in rodents. The protective effect is observed when squalene is given before and/or during the treatment of carcinogen. The underlying mechanisms of squalene for the prevention of cancer may include ras farnesylation inhibition, carcinogen activation modulation and anti-oxidative activities [41].

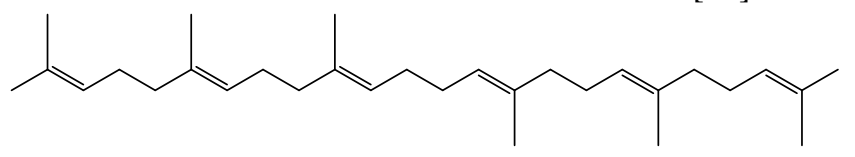

Fig.9. $(6 E, 10 E, 14 E, 18 E)-2,6,10,15,19,23$-hexamethyltetracosa$2,6,10,14,18,22$-hexaene 


\section{Indo Global Journal of Pharmaceutical Sciences, 2019; 9(1): 32-41}

Quercetin: Quercetin is a plant-derived flavonoid, used as nutritional supplement, found in apple skins, berries, brassica vegetables, broccoli, brussel sprouts, cereals, cherries, and cauliflower, grape juice, kohlrabi, oregano, pears, peppers, red and yellow onions, tea, tomatoes leaves, and wine [Fig 10]. Quercetin, like parsely can also help to retard the activity of the enzymes that can cause cancer growth and spread it. It is the strongest of nature's anti-inflammatory drugs [42]. Quercetin also shows anti-tumour properties. Skin and prostate cancers show $90 \%$ mortality within 48 hours with no deteriorating effect on surrounding normal cells when quercetin is co-administered with ultrasound at $20 \mathrm{kHz}$ for 1 minute [43]. Also quercetin aglycone has been shown to interact with aryl hydrocarbon receptors, which are primarily involved in the development of certain cancers induced by chemicals. The signal transduction pathways involving MEK/ERK and Nrf2/keap1, which are responsible for development of inflammation and carcinogenesis are modulated by quercetin aglycone [44].

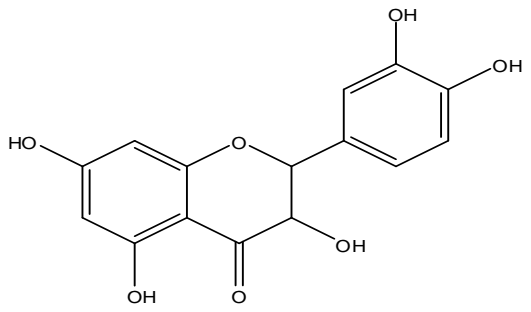

Fig.10. 2-(3,4- dihydroxyphenyl)- 3,5,7- trihydroxy- 4Hchromen- 4-one

Artimisinin: Artimisinin, a sesquiterpene lactone, obtained from sweetworm wood has been used for decades for fever and shivering. Though the antimalarial activity of artimisinin is well known, moreover, its antiviral, antifungal, antiinflammatory and anticancer activity has also been reported. Artimisinin mediated cytotoxicity was related to the induction of caspase dependent and mitochondrial pathway mediated apoptosis including oxidative stress, endoplasmic reticulum stress and DNA damage that lead to apoptotic cell death [45].

Vitamin C: Vitamin C is found in broccoli, cabbage, citrus fruits (grapefruit, lemons, oranges), cranberries, leafy green vegetables (kale, spinach), melons, peppers, potatoes, strawberries, and tomatoes. Vitamin $\mathrm{C}$ can prevent the damage caused by oxygen free radicals by mutation and may lead to the occurence and proliferation of cancer in healty cells [46].

Vitamin D: Vitamin D is a group of fat-soluble secosteroids, Vitamin D2 (ergocalciferol) and Vitamin D3 (cholecalciferol) are the two major physiologically important forms of vitamin D. It helps in production of enzymes called phosphotases, which are involved in de-activation of other enzymes called kinases, essential to growth and reproduction of cancer cells. Polymorphisms of the vitamin D receptor (VDR) gene have been associated with an increased risk of breast cancer. Women with mutations in the VDR gene had an increased risk of breast cancer [47]. Breast cancer disease progression and bone metastases are also associated with low levels of vit $\mathrm{D}$ [48].

\section{REGULATORY SCENARIO OF NUTRACEUTICALS}

Presuming the safety and therapeutic efficacy of Nutraceuticals, they are given due importance, in today's scenario. Inspite of unremarkable progress in use of nutraceuticals, there are still several issues including clinical evidence supporting in vitro claims, regulatory aspects and assurance of nutraceuticals identity, quality and safety which needs to be considered [49]. There is a need of regulating or categorizing these food products under specific definitions and according to food laws. The primary regulatory obligations of manufacturers and marketers of nutraceuticals include product safety and accuracy of the labeling that specify product identity and composition. The food sector in India is not governed by a single comprehensive body but by a no of laws and regulations that has led to complex conflicting and overlapping rules. But after a much sought out decision and following the pressure from food industry, Govt of India gave way to a single regulatory body with modern comprehensive laws, the Food Safety and Standard Act,2006 (FSSA). The FSSA is a ladder that steps towards bringing a transformational shift in the food regulatory scenario of the country and also aims to establish a single reference point for all matters in relation to food safety and standards, by moving from multi-level control system to a single integrated one. FSSA has recognized nutraceuticals as functional foods and categorized them under foods, but the rule and regulations are yet to be framed. The emerging regulations must ensure that nutraceuticals are regulated in the most efficient manner that maximizes health benefits and minimizes health hazard for users and genuiness of the claims that are made by the firms producing and selling them [50].

\section{CONCLUSION}

Cancer is a deadly disease and complexities of disease are such that no single available anticancer agent can satisfactory combat the multiple disorders of the disease. Therefore, there is an urgent need to search for better medicines for cancer. In this context, nutraceuticals are promising candidates to treat cancer. Most of the plants and herbs as mentioned are used in remote villages and tribal pockets as folk medicine and are not 


\section{Indo Global Journal of Pharmaceutical Sciences, 2019; 9(1): 32-41}

known to the mainstream population. Nutraceuticals, an entirely new segment of the health industry has been predicted to be the next financial blockbuster. Nutraceuticals are the next generation of food supplements that bridge the gap between supplements and pharmaceuticals .Undoubtedly, nutraceuticals have provided useful drugs to mankind for their health care and other needs. The effort to combat the diseases, for which there is no satisfactory solution as yet, should be continued relentlessly. Hopefully, plant kingdom constituting miraculous herbs or so called sanjivini bootis of today will help us in the discovery of new drug products useful for the alleviation of deadly human diseases like cancer.

\section{REFERENCES}

1. Santini, A., Novellino, E. Nutraceuticals Food Supplements \& Cholesterol Level Control. Annals of Pnarmacology \& Pharmaceutics., 2017; 2 (19): 1099.

2. Sut, S., Baldan, V., Faggian, M., Peron, G., Dall, A.S. Nurtraceuticals, A new Challenge for Medicinal Chemistry. Curr Med Chem., 2016; 23(28): 3198-3223.

3. Lauren, P. Molecular Biology of Cancer: Mechanisms, Targets, and Therapeutics, 2nd Edition; Oxford University Press, 2008.

4. Kokate, CK., Purohit, A.P., Gokhale, S.B. Textbook of Pharmacognosy, 51st Edition; Nirali Prakashan, 2015.

5. Powolny, A.A., Singh, S.V. Multitargeted prevention and therapy of cancer by diallyl trisulfide and related allium vegetable-derived organosulfur compounds. Cancer Lett., 2008 ; 269 (2): 305-314.

6. Lau, M.D. et al. Allium sativum (Garlic) and cancer prevention. Nutrition research, 1990; 10 (8): 937-948.

7. Mignone, L.I., Giovannucci, E., Newcomb, P.A. Dietary carotenoids and the risk of invasive breast cancer. Int. J. Cancer, 2009; 124 (12): 2929-37.

8. Zhao, C., Giusti, M.M., Malik, M., Moyer, M.P., Magnuson, B.A. Effects of commercial anthocyanin-rich extracts on colonic cancer and non tumourigenic colonic cell growth. J. Agric. Food Chem., 2004; 52 (20): 6122-8.

9. Wang, L.S., Stoner, G.D. Anthocyanins and their role in cancer prevention. Cancer Lett., 2008; 269 (2): 281-290.

10. Leiro, J. et al. Effect of cis-resveratrol on genes involved in nuclear factor kappa B signaling. Int. Immunopharmacol., 2005; 5 (2): 393406.

11. Chun, Y.J, Kim, M., Guengerich, F.P. Resveratrol is a selective human cytochrome P450 1A1 inhibitor. Biochem. Biophys. Res. Commun., 1999; 262 (1): 20-24.

12. Omenn G.S. et al. Effects of a combination of beta carotene and vitamin A on lung cancer and cardiovascular disease. N. Engl. J. Med., 1996; 334 (18):1150-1155.

13. Russel, R.M. Beta-carotene and lung cancer. Pure Appl. Chem., 2002; 74 (8):1461-1467.

14. Awad, A.B., Chen, Y.C, Fink, C.S., Hennessey, T. Beta-sitosterol inhibit HT-29 human colon cancer cell growth and alter membrane lipids. Anticancer Res., 1996; (16):2797-804.

15. Evans, C.A., Miller, N.J. Antioxidant activities of flavonoids as bioactive components of food. Biochem. Soc. Trans., 1996; 24 (3):790795.

16. Chung, T.W. et al. Novel and therapeutic effect of caffeic acid and caffeic acid phenyl ester on hepatocarcinoma cells: complete regression of hepatoma growth and metastasis by dual mechanism. FASEB J., 2004; 18 (14):1670-81.

17. Marimuthu, S., Adluri, S.R., Venugopal, M.P. Ferulic Acid: Therapeutic Potential through Its Antioxidant Property. J. Clin. Biochem. Nutr., 2007;40 (2):92-100.

18. Ito, K. et al. Induction of apoptosis in leukemic cells by homovanillic acid derivative, capsaicin, through oxidative stress: implication of phosphorylation of p53 at Ser-15 residue by reactive oxygen species. Cancer Res., 2004; 64 (3):1071-1078.
19. Oyagbemi, A.A., Saba, A.B., Azeez, O.I. Capsaicin: A novel chemopreventive molecule and its underlying molecular mechanisms of action. Indian J. Cancer., 2010; 47 (1): 53-58.

20. Pathak, A.K. et al. Ursolic acid inhibits STAT3 activation pathway leading to suppression of proliferation and chemo sensitization of human multiple myeloma cells. Mol. Cancer Res., 2007; 5 (9): 943-55.

21. Huang, M.T., Ho, C.T., Wang, Z.Y., Ferraro, T. Inhibition of skin tumorigenesis by rosemary and its constituents carnosol and ursolic acid. Cancer Res., 1994; 54 (3):701-8.

22. Chen, D., Dou, Q.P. Tea polyphenols and their roles in cancer prevention and chemotherapy. Int. J. Mol. Sci., 2008; 9 (7):1196-206.

23. Shahidi, F. Nutraceuticals functional foods and dietary supplements in health and disease. Journal of Food and Drug Analysis., 2012; 20: 226230 .

24. Angelo, L.S., Kurzrock, R. Turmeric and green tea: a recipe for the treatment of B- chronic lymphocytic leukemia. Clin. Cancer Res., 2009; 15 (4): 1123-5.

25. Ravindran, J., Prasad, S., Aggarwal, B. Curcumin and Cancer Cells: How Many Ways Can Curry Kill Tumour Cells Selectively? The AAPS journal., 2009; 11 (3): 495.

26. Aggarwal, B.B., Shishodia, S. Suppression of the nuclear factorkappaB activation pathway by spice-derived phytochemicals: reasoning for seasoning. Ann. NY. Acad. Sci., 2004; 1030: 434-441.

27. Switzer, C.H. et al. Dithiolethione compounds inhibit Akt signaling in human breast and lung cancer cells by increasing PP2A activityACS-1 increases PP2A activity. Oncogene., 2009; 28: 3837-3846.

28. Singh, S.V. et al. Novel Anti-Carcinogenic Activity of an Organosulfide from Garlic: Inhibition of H-RAS Oncogene Transformed Tumour Growth in vivo by Diallyl Disulfide Is Associated with Inhibition of p21H-rasProcessing, Biochem. Biophys. Res. Commun., 1996; 225 (2):660-665.

29. Chen, Y.Q. et al. Modulation of prostate cancer genetic risk by omega3 and omega-6 fatty acids. J. Clin. Invest. 2007; 117 (7):1866-75.

30. Pala, V. et al. Erythrocyte Membrane Fatty Acids and Subsequent Breast Cancer: A Prospective Italian Study, JNCL., 2001; 93 (14): 1088 .

31. Rhode, J., Fogoros, S., Zick, S. Ginger inhibits cell growth and modulates angiogenic factors in ovarian cancer cells. BMC Complement Altern. Med., 2007; 7:44.

32. Jeong, C.H. et al. Gingerol Suppresses Colon Cancer Growth by Targeting Leukotriene A4 Hydrolase. Cancer Res., 2009 69; (13):5584-91.

33. Sarkar, F.H., Li, Y. Soy isoflavones and cancer prevention. Cancer Invest. 2003; 21(5): 817-8.

34. Kaji, I. et al. Inhibition by d-limonene of experimental hepato carcinogenesis in Sprague-Dawley rats does not involve p21 (ras) plasma membrane association. Int. J. Cancer., 2001; 93 (3): 441-4.

35. Uedo $\mathrm{N}$. et al. Inhibition by d-limonene of gastric carcinogenesis induced by N-methyl-N'-nitro-N-nitrosoguanidine in Wistar rats. Cancer Lett., 1999;137 (2): 131-136.

36. Nwanodi, O.B. Nutraceuticals: Curative integrative cancer treatment. Alternative and Integrative Medicine. 2017; 6 (2): 1-12.

37. Bonnesen, C., Eggleston, I.M., Hayes, J.D. Dietary indoles and isothiocyanates that are generated from cruciferous vegetables can both stimulate apoptosis and confer protection against DNA damage in human colon cell lines. Cancer Res., 2001; 61 (16):6120-6130.

38. Sun, H.X., Xie, Y., Ye, YP. Advances in saponin-based adjuvants, Vaccine., 2009: 27(12):1787-96.

39. Facompre, N., El-Bayoumy, K. Potential Stages for Prostate Cancer Prevention with Selenium: Implications for Cancer Survivors. Cancer Res., 2009; 69 (7):2699-2703.

40. Klein, E.A. et al. SELECT: the selenium and vitamin E cancer prevention trial: rationale and design. Prostate Cancer Prostatic Dis., 2000; 3: 145-51.

41. Sotiroudis, T.G., Kyrtopoulos, S.A. Anticarcinogenic compounds of olive oil and related biomarkers. Eur. J. Nutr., 2008; 47 (2): 69-72.

42. Hirpara, K.V., Aggarwal, P., Mukherjee, A.J., Joshi, N., Burman, A.C. Quercetin and its derivatives: synthesis, pharmacological uses with special emphasis on anti-tumor properties and prodrug with enhanced bio-availability. Anti-cancer Agents Med. Chem, 2009; 9 (2):138-61.

43. Paliwal, S., Sundaram, J., Mitragotri, S. Induction of cancer-specific cytotoxicity towards human prostate and skin cells using quercetin and ultrasound. Br. J .Cancer., 2005;92 (3):499-502. 
Indo Global Journal of Pharmaceutical Sciences, 2019; 9(1): 32-41

44. Murakami, A., Ashida, H., Terao, J. Multi targeted cancer prevention by quercetin, Cancer Lett., 2008;269 (2): 315-325.

45. Wong, Y.K. et al. Artemisinin as an anticancer drug: Recent advances in target prolifing and mechanisms of action. Med. Res. Rev. 2017; 37: $1492-1517$.

46. Traber, M.G., Frei, B. Vitamins $\mathrm{E}$ and $\mathrm{C}$ in the Prevention of Cardiovascular Disease and Cancer in Men. Free Radic Biol Med., $2009 ; 300$ (18):2123-2133.

47. Buyru, N., Tezol, A., Yosunkaya F.E., Dalay, N. Vitamin D receptor gene polymorphisms in breast cancer., Exp. Mol. Med., 2003;35 (6) :550-555.

48. Garland, C.F. et al. The role of vitamin D in cancer prevention, Am. J. Public Health., 2006; 96 (2): 252-61.

49. Estevinho, L.M. Nutraceuticals in human health and disease. International Journal of Molecular Sciences. Editorial- special issue, 2018; $1-3$.

50. Palthur, M.P., Palthur, S., Sajala, S., Chitta, S.K. Nutraceuticals: Concept and regulatory scenario, Int. J. Pharm. Pharm. Sci., 2010; 2: $14-20$.

Indo Global Journal of Pharmaceutical Sciences( ISSN 2249 1023; CODEN- IGJPAI; NLM ID: 101610675) indexed and abstracted in CrossRef (DOI Enabling), UGC CARE Journal List, EMBASE(Elsevier), National Library of Medicine (NLM) Catalog, ResearchGate, Publons, CAS (ACS), Index Copernicus, Google Scholar and many more. For further details, visit http://iglobaljournal.com 